\title{
Formation of Hollow Nanocrystals through the Nanoscale Kirkendall Effect
}

\author{
Yadong Yin, Robert M. Rioux, Can K. Erdonmez, Steven Hughes, Gabor A. \\ Somorjai, A. Paul Alivisatos*
}

Department of Chemistry, University of California at Berkeley, and Materials Science Division, Lawrence Berkeley National Laboratory, Berkeley, CA 94720, USA.

*To whom correspondence should be addressed.Email: alivis@uclink4.berkeley.edu

\begin{abstract}
We demonstrate that hollow nanocrystals can be synthesized through a mechanism analogous to the Kirkendall Effect, in which pores form due to the difference in diffusion rates between two components in a diffusion couple. Cobalt nanocrystals are chosen as a primary example to show that their reaction in solution with oxygen, sulfur or selenium leads to the formation of hollow nanocrystals of the resulting oxide and chalcogenides. This process provides a general route to the synthesis of hollow nanostructures of large numbers of compounds. A simple extension of this process yields platinum-cobalt oxide yolk-shell nanostructures which may serve as nanoscale reactors in catalytic applications.
\end{abstract}


Porous solid materials are important in many areas of modern science and technology, including ion exchange, molecular separation, catalysis, chromatography, microelectronics, and energy storage (1-3). Notable examples are microporous $(<2 \mathrm{~nm})$ zeolites and mesoporous $(2-50$ nm) silicate and carbonaceous materials. The ability to manipulate the structure and morphology of porous solids on a nanometer scale would enable greater control of local chemical environment (4-6). Here, we demonstrate that nanoscale pores can develop inside nanocrystals with a mechanism analogous to void formation in the Kirkendall Effect, where the mutual diffusion rates of two components in a diffusion couple differ by a considerable amount (7). We choose cobalt nanocrystals as a starting material to show that hollow nanocrystals of cobalt oxide and chalcogenides can be successfully synthesized by reacting cobalt colloidal solution with oxygen, sulfur or selenium. This principle enables the synthesis of hollow structures of large numbers of compounds. We also demonstrate that the resulting hollow nanocrystals can encapsulate catalyst particles to form novel nanoscale reactors.

It has been known for over half a century that porosity may result from differential solidstate diffusion rates of the reactants in an alloying or oxidation reaction. In 1947, Smigelkas and Kirkendall reported the movement of the interface between a diffusion couple, i.e., copper and zinc in brass, as the result of the different diffusion rates of these two species at an elevated temperature (7). This phenomenon, now called the Kirkendall Effect, was the first experimental proof that atomic diffusion occurs through vacancy exchange, not the direct interchange of atoms. The net directional flow of matter is balanced by an opposite flow of vacancies, which can condense into pores or annihilate at dislocations. Directional material flows also result from coupled reaction-diffusion phenomena at a solid/gas or solid/liquid interface, leading to deformation and/or void formation during the growth of metal oxide or sulfide films $(8,9)$. 
These voids are usually explained by outward transport of fast-moving cations through the oxide layer and a balancing inward flow of vacancies to the vicinity of the metal-oxide interface. Interface motion and the formation of pores have been studied due to their impact on the reproducibility or reliability of solders, passivation layers, diffusion barriers etc., but not generally as a method of preparing porous materials. The pores produced at a metal-metal diffusion couple or near the metal-oxide interface of a growing oxide do not yield monodisperse, ordered arrays but form a very heterogeneous ensemble. Also, the observed volume fraction for pores is commonly much smaller than would be expected for the known material flows. These observations are a direct result of the large volume of material that vacancies can diffuse into and the large number of defects they can react with (10).

If the faster-diffusing species is confined into a nanocrystal 'core', the net rate of vacancy injection should increase dramatically due to the high surface-to-volume ratio of the particle and the absence of defects in the core. Within the small volume of a transforming nanocrystal, the supersaturated vacancy cloud is likely to coalesce into a single void. Previous studies on the interdiffusion of 30-micrometer powders with layered composition showed significant porosity, but the geometry and distribution of the pores were not uniform, probably due to aggregation and still bulk-like dimension of the particles (11). Recently, significant progress has been made in synthesizing colloidal nanocrystals with well-controlled size, shape and surface properties (1214). Employing such high-quality nanocrystals as the starting materials, it should be possible to produce a uniform population of hollow nanostructures.

We have chosen Co nanocrystals as the main starting material. A number of chemical methods have been developed to synthesize uniform Co nanocrystals in solution $(12,15)$. Furthermore, cobalt reacts readily with other species such as sulfur and oxygen. Since cobalt is 
the major component in one class of superalloys, its high-temperature oxidation and sulfidation have been well studied $(16,17)$. It is known that oxidation and sulfidation of bulk cobalt under vapor at high temperature is mainly controlled by outward diffusion of cobalt cations (18). This mode of growth operating on nanocrystals is expected to lead to hollow structures.

Sulfidation of cobalt was the first case where we observed hollow nanostructures. Cobalt sulfide hollow nanospheres are synthesized in one pot by immediate injection of a solution of sulfur in $o$-dichlorobenzene into a hot Co nanocrystal dispersion (as shown in the transmission electron microscopy (TEM) image in Fig. 1A) that is prepared by literature methods $(15,19)$. At $445 \mathrm{~K}$, the reaction between cobalt and sulfur completes within a few seconds, resulting in a stable black solution of cobalt sulfide nanocrystals. We have confirmed that hollow particles are produced at temperatures as low as $373 \mathrm{~K}$. Apparently, the chemical transformation of the surface does not disrupt the stabilization of the nanocrystals by surfactant molecules.

Outward flow of cobalt through the sulfide shell results in supersaturation of vacancies, which condense to form a single hole in each nanoparticle (Figs. 1B-D). Two stable cobalt sulfide phases are observed, linnaeite $\left(\mathrm{Co}_{3} \mathrm{~S}_{4}\right)$ and cobalt pentlandite $\left(\mathrm{Co}_{9} \mathrm{~S}_{8}\right)$, depending on the sulfur-to-cobalt molar ratio used in the synthesis. X-ray powder diffraction (XRD) patterns in Fig. 1E show the evolution of the crystal structure as the molar ratio of sulfur to cobalt is increased. $\mathrm{Co}_{9} \mathrm{~S}_{8}$ is the only sulfide phase observed when the molar ratio is lower than $9: 8$, while $\mathrm{Co}_{3} \mathrm{~S}_{4}$ also appears in the patterns when the molar ratio slightly exceeds this value. Only $\mathrm{Co}_{3} \mathrm{~S}_{4}$ is obtained when the molar ratio of sulfur to cobalt is above $3: 4$. The size distribution of the sulfide hollow particles is similar to the starting cobalt nanocrystals. Monodisperse hollow nanocrystals self-assemble into ordered hexagonal arrangements when evaporated slowly on the surface of a carbon coated TEM grid. The assembly process is driven by surface tension and van der Waals 
forces. Cobalt sulfide nanocrystals do not form superlattices as readily as cobalt nanocrystals do, probably due to a diminished van der Waals force and the absence of magnetic interactions among sulfide particles (19). Assemblies of hollow nanoparticles present a distinct topology of ordered porous materials. In terms of the accessibility of pores from the outside, they fall between mesoporous materials with accessible channels and void lattices where pores are confined in a continuous matrix (20).

Kinematical diffraction simulations indicate that the XRD peaks are too broad to be consistent with a single crystal shell of dimensions observed by TEM (21). A satisfactory fit to the data in Fig. $1 \mathrm{E}(\mathrm{d})$ is obtained by assuming a $4.5 \mathrm{~nm}$ cubic crystalline domain. TEM micrographs (Fig. 1D) of the same sample show that the average outer diameter of the hollow $\mathrm{Co}_{9} \mathrm{~S}_{8}$ nanocrystals is around $15 \mathrm{~nm}$. A reasonable explanation is that the shell of each hollow sphere is multi-crystalline. This is confirmed by high resolution transmission electron microscopy (HRTEM). As shown in Fig. $1 \mathrm{C}$, both $\mathrm{Co}_{9} \mathrm{~S}_{8}$ and $\mathrm{Co}_{3} \mathrm{~S}_{4}$ hollow nanocrystals are composed of multiple crystallographic domains. The arrangement of the domains is analogous to the columnar morphology of grains often observed in thin film growth. The multi-crystalline structure implies possible applications of these hollow nanocrystals as nanoscale reactors since small molecules may be able to penetrate the shell through the grain boundaries.

In all instances of sulfidation, we have found that the diameter of the hole in the center of the nanocrystals is $40-70 \%$ of the initial particle size (starting with Co particles with a size distribution of $7 \%$, a single synthesis yields a hole size distribution of $13 \%$ ). If sulfur transport through the growing shell is negligible, as shown for bulk sulfidation by marker experiments (18), then the two diameters are expected to be identical. Significant inward sulfur transport could occur through grain boundaries or during the formation of the first few monolayers of sulfide. It 
is also possible that inward relaxation of the hole occurs, due to annihilation of vacancies at a semicoherent or incoherent cobalt-sulfide interface. Finally, the estimation of the hole size by visual inspection of TEM images may produce systematic errors. We attempted to examine the possibility of inward sulfur transport by performing the $\mathrm{Co}_{3} \mathrm{~S}_{4}$ synthesis at different sulfur concentrations. Increased sulfur concentration increases hole size and enhances outward growth of the shell, indicating that cobalt mobility rather than sulfur mobility is affected. This finding is consistent with bulk sulfidation studies (18), where it is observed that an increased sulfur vapor pressure leads to injection of more cation vacancies into the growing sulfide and enhances the parabolic rate constant for sulfide growth.

For bulk cobalt, the rates of oxidation are 3-4 orders of magnitude lower than those of sulfidation above $750 \mathrm{~K}(18)$. This is also true under the conditions we use to produce hollow nanocrystals, and oxidation of nanocrystals takes about three hours at $455 \mathrm{~K}$. Fig. 2(A-D) shows the evolution of the morphology of the nanocrystals with time as an $\mathrm{O}_{2} / \mathrm{Ar}$ mixture is flowed through the cobalt colloidal solution. The XRD shows the presence of metallic cobalt up to 30 minutes (Fig. 2E). The solution of particles still displays weak ferrofluidic response to a $1 \mathrm{~T}$ magnet at that point, suggesting that small cobalt cores remain. It takes about three hours for the cobalt cores to be completely consumed: central pores are clearly distinguishable for all nanocrystals under TEM and the solution shows no magnetic response.

The evolution of hollow morphology is best illustrated by following the reaction of cobalt nanocrystals with selenium. In bulk systems, annihilation of excess vacancies at dislocations and boundaries can produce stresses leading to the formation of cracks near the interface; the cracks then act as nuclei for the further condensation of supersaturated vacancies (11). While the exact mechanism is likely to be different, in nanocrystals voids also begin to develop and merge at the 
boundary (Fig. 3). The high defect content and surface energy associated with the boundary favors the nucleation of voids there. Also, as vacancies diffuse inwards, they will be more concentrated at the boundary rather than in the interior of the core. As the reaction proceeds in time, more cobalt atoms diffuse out to the shell and the accompanying transport of vacancies leads to growth and merging of the initial voids. This results in the formation of bridges of material between the core and the shell that persist until the core is completely consumed. These bridges provide a fast transport path for outward diffusion of cobalt atoms which can then spread on the inner shell surface. A similar phenomenon was observed for bulk powders in Ref. 11. We note that the growth rate of pores drops dramatically when the cobalt cores become relatively small. Most of the pore volume seems to form during the first few minutes, while it takes about 30 minutes for the cobalt cores to completely disappear. This may be due to the fact that as the bridges are also consumed during the reaction, a smaller cross-sectional area is available for solid-state transport of materials.

As an illustration of the generality of the ideas presented here, we have synthesized several other hollow nanostructures. Sulfidation of disk-shaped Co nanocrystals (21) was observed to result in the formation of hollow nanodisks with cylindrical pores, indicating that spherical symmetry is not required for obtaining shells of regular thickness. Preliminary studies on oxidation of iron nanospheres and sulfidation of cadmium nanospheres also resulted in hollow structures, thus validating our approach for metallic cores in general. Theoretically, the mobilities of the reacting species do not have to be drastically different to result in vacancy transport. Placing solid nanocrystals containing one reactant in a comparatively dilute solution creates an additional asymmetry that may favor the creation of hollow structures: the relatively large change in the concentration of the core material between the core and the solution provides 
a greater driving force for the outward diffusion of the core material. Thus, numerous combinations of reactants may be expected to produce various hollow nanostructures of insulators, semiconductors and even metals. A recent report on the formation of gold nanoboxes may involve the same mechanism at some stage, although the dimension of the structures produced is an order of magnitude larger (22).

Hollow nanocrystals offer exciting possibilities in material design for applications in catalysis, nanoelectronics, nano-optics, drug delivery systems, and as building blocks for lightweight structural materials (23-25). For example, accurate fixation of the catalyst within the pores, combined with other emerging techniques of chemical control (26), could result in better reaction control and new products. To demonstrate the use of hollow nanocrystals in catalysis, we study their function as nanoreactors each containing one noble metal nanocrystal. A Pt@CoO yolk-shell nanostructure is synthesized, in which a Pt nanocrystal of a few nanometers is encapsulated in a $\mathrm{CoO}$ shell. Three steps are involved in the preparation of these nanoreactors: synthesis of Pt seeds by a modified "polyol" process (27), deposition of cobalt on Pt to form Pt@Co core-shell nanocrystals, and transformation of Co into CoO hollow structures (28). Fig. 4A shows a typical sample of Pt particles with an average diameter around $3 \mathrm{~nm}$. The deposition of Co onto Pt at the reaction temperature yields no alloy, only Pt core/Co shell particles, as confirmed by XRD analyses. The oxidation reaction removes Co atoms away from the $\mathrm{Pt}$ particle surface, leading to the formation of a $\mathrm{Pt}$ yolk/CoO shell structure (Fig. 4B). No free platinum particles were found by TEM inspections on the Pt@CoO sample. The final size of Pt@CoO particles can be controlled by changing the diameter and number of the Pt seeds, and the amount of cobalt carbonyl precursor. 
In order to determine if the $\mathrm{Pt} @ \mathrm{CoO}$ materials were active as heterogeneous catalysts, the hydrogenation of ethylene was chosen as a model reaction since it readily occurs at ambient conditions on many transition metal catalysts. Platinum is one of the most active metals for this reaction while the activity of metallic cobalt is approximately two orders of magnitude lower (29). We found that pure CoO hollow nanocrystals are inactive for ethylene hydrogenation (30), even following a $1 \mathrm{~h} \mathrm{H}_{2}$ pre-reduction at $373 \mathrm{~K}$. Only upon reduction at $473 \mathrm{~K}$ for $1 \mathrm{~h}$ is ethane detected at temperatures $>300 \mathrm{~K}$. Samples containing Pt are active for $\mathrm{C}_{2} \mathrm{H}_{4}$ hydrogenation at temperatures as low as $208 \mathrm{~K}$, with samples subjected to no pretreatment. The steady state turnover frequency for ethane formation at $227 \mathrm{~K}$ is $8.3 \times 10^{-3} \mathrm{~s}^{-1}(31)$, which is comparable to rate of $3.5 \times 10^{-2} \mathrm{~s}^{-1}$ measured on a $0.04 \% \mathrm{Pt} / \mathrm{SiO}_{2}$ catalyst (32). Comparison of reaction rates (on per gram total catalyst basis) at $300 \mathrm{~K}$ demonstrate that $\mathrm{Pt} @ \mathrm{CoO}$ is 2 orders of magnitude more active than $\mathrm{CoO}$. These observations indicate that the reaction is catalyzed by Pt particles, not the $\mathrm{CoO}$ shell. This also confirms that a route exists for ethylene and hydrogen entry into the $\mathrm{CoO}$ shell interior. The grain boundaries on the shell are the most probable entry points for ethylene and hydrogen diffusion into as well as ethane diffusion out of the shell.

There may be several unique catalytic applications of such hollow nanocrystals if we place a transition metal (such as platinum) nanoparticle inside the nanocavity. 1) At low temperatures, platinum can carry out hydrocarbon conversion reactions without any participation of the cobalt oxide shell. These reactions include hydrogenation, dehydrogenation of ethylene and cyclohexene or the isomerization of $\mathrm{C}_{6}$ hydrocarbons such as 1-hexene, 2-methyl and 3methylpentanes. The complete separation of the Pt nanoparticles by the cobalt oxide shell prevents secondary reaction of the products that would change selectivity and desired product distribution. 2) At higher reaction temperatures, both the cobalt compound and the $\mathrm{Pt}$ 
nanoparticle inside the core are active. In this circumstance, the reaction selectivity can be altered because $\mathrm{Pt}$ readily atomizes $\mathrm{H}_{2}$ or $\mathrm{O}_{2}$ to provide superior reducing or oxidizing power for the cobalt compound. It is hoped that the core structure maintains its structural stability in this circumstance. Reactions of interest include CO hydrogenation, partial oxidation of light alkanes and hydrodesulfurization of thiophene.

References:

1. D. Zhao, P. Yang, Q. Huo, B. F. Chmelka, G. D. Stucky, Curr. Opin. Solid State Mater. Sci. 3, $111(1998)$.

2. S. A. Johnson, P. J. Ollivier, T. E. Mallouk, Science 283, 963 (1999).

3. A.-P. Li, F. Müller, A. Birner, K. Nielsch, U. Gösele, Adv. Mater. 11, 483 (1999).

4. D. Trong On, D. Desplantier-Giscard, C. Danumah, S. Kaliaguine, Appl. Catal. 222, 299 (2001).

5. M. E. Davis, Nature 417, 813 (2002).

6. W. Gu, M. Warrier, V. Ramamurthy, R. G. Weiss, J. Am. Chem. Soc. 121, 9467 (1999).

7. A. D. Smigelskas, E. O. Kirkendall, Trans. AIME 171, 130 (1947).

8. C. E. Birchenall, J. Electrochem. Soc. 103, 619 (1956).

9. J. C. Colson, M. Lambertin, P. Barret in Proc. 7th Int. Symp. Reactivity of Solids, J. S. Anderson, F. S. Stone, M. W. Roberts Eds. (Chapman and Hall, London, 1972), pp. 283-293.

10. G. B. Gibbs, Oxid. Met. 16, 147 (1981).

11. F. Aldinger, Acta Met. 22, 923 (1974).

12. C. B. Murray, C. R. Kagan, M. G. Bawendi, Annu. Rev. Mater. Sci. 30, 545 (2000). 
13 X. Peng, L. Manna, W. Yang, J. Wickham, E. Scher, A. Kadavanich, A.P. Alivisatos, Nature 404, 59 (2000)

14. R. Jin, Y. C. Cao, E. Hao, G. S. Metraux, G. C. Schatz, C. A. Mirkin, Nature 425, 487 (2003).

15. V. F. Puntes, K. M. Krishnan, A. P. Alivisatos, Science 291, 2115 (2001).

16. S. Mrowec, K. Przybylski, Oxid. Met. 11, 365 (1977).

17. A. Devin, Cobalt 30, 19 (1966).

18. S. Mrowec, M. Danlelewski, A. Wojtowicz, J. Mater. Sci. 33, 2617 (1998).

19. V. F. Puntes, K. M. Krishnan, P. Alivisatos, Appl. Phys. Lett. 78, 2187 (2001).

20. N. M. Ghoniem, D. Walgraef, S. J. Zinkle, J. Comput. Aided Mater. Des. 8, 1 (2001).

21. V. F. Puntes, D. Zanchet, C. K. Erdonmez, A. P. Alivisatos, J. Am. Chem. Soc. 124, 12874 (2002).

22. Y. Sun, Y. Xia, Science 298, 2176 (2002).

23. F. Caruso, R. A. Caruso, H. Möhwald, Science 282, 1111 (1998).

24. U. S. Schwarz, S. A. Safran, Phys. Rev. E 62, 6957 (2000).

25. W. S. Sanders, L. J. Gibson, Mater. Sci. Eng. A 352, 150 (2003).

26. N. J. Turro, Acc. Chem. Res. 33, 637 (2000).

27. N. S. Sobal, U. Ebels, H. Möhwald, M. Giersig, J. Phys. Chem. 107, 7351 (2003).

28. Platinum acetylacetonate is reduced with a long-chain polyol to form uniform Pt nanoparticles in the presence of surfactants such as oleic acid, oleylamine, and trioctylphosphine. The size of the Pt particles can be tuned from 1 to $10 \mathrm{~nm}$, depending on the concentration of surfactants. $\mathrm{Co}_{2}(\mathrm{CO})_{8}$ is then injected into the hot solution and decomposed to form a conformal coating on Pt nanocrystals. Oxidation of the Pt@Co nanocrystals is performed a few minutes after the introduction of cobalt carbonyl by blowing a stream of $\mathrm{O}_{2} / \mathrm{Ar}$ (1:4 in volume ratio, 120 
$\mathrm{ml} / \mathrm{min}$ ) mixture into the colloidal solution at $455 \mathrm{~K}$. The system is kept under stirring for 3 hours. A black stable colloidal dispersion in o-dichlorobenzene is obtained. Finally, the Pt@CoO particles are precipitated by methanol, washed with toluene and methanol three times, and dried under vacuum. Typical nitrogen adsorption/desorption measurement on the powder at $77 \mathrm{~K}$ shows a type IV isotherm with type $\mathrm{H} 2$ hysteresis, with a Brunauer-Emmet-Teller (BET) surface area of $65 \mathrm{~m}^{2} / \mathrm{g}$ and a total pore volume of $0.0676 \mathrm{~cm}^{3} / \mathrm{g}$.

29. G. A. Somorjai, Introduction to Surface Chemistry and Catalysis. (John Wiley and Sons, Inc., New York, 1994).

30. The hydrogenation of ethylene is studied at atmospheric pressure in a differentially operated plug flow reactor. Standard conditions are 11 Torr $\mathrm{C}_{2} \mathrm{H}_{4}, 150$ Torr $\mathrm{H}_{2}$, and 208-353K (sample dependent).

31. Rates were measured on a per gram basis. They were normalized per mole of surface Pt species $\left(\mathrm{Pt}_{\mathrm{s}}\right.$ ) to obtain a turnover frequency (molecule $\mathrm{Pt}_{\mathrm{s}}{ }^{-1} \mathrm{~S}^{-1}$ ). Moles of $\mathrm{Pt}_{\mathrm{s}}$ was determined by $\mathrm{D}=1.13 / \mathrm{d}$ where $\mathrm{D}$ is the $\mathrm{Pt}$ dispersion (ratio of $\mathrm{Pt}_{\mathrm{s}}$ to the total $\mathrm{Pt}$ content $\left(\mathrm{Pt}_{\mathrm{t}}\right)$ and $\mathrm{d}$ is the $\mathrm{Pt}$ particle size in $\mathrm{nm}$. The $\mathrm{Pt}$ particle size was determined from number average TEM measurements.

32. R. D. Cortright, S. A. Goddard, J. E. Rekoske, J. E. Dumesic, J. Catal. 127, 342 (1991).

33. This work was supported by the Air Force Office of Scientific Research under award number F49620-01-1-0033, and by the Director, Office of Energy Research, Office of Science, Division of Materials Sciences, of the U.S. Department of Energy under Contract No. DE-AC0376SF00098. Y.Y. thanks Prof. J. Fréchet for the valuable discussions. R.M.R. would like to acknowledge the Ford Motor Company and the Berkeley Catalysis Center for financial support. 


\section{Captions to Figures}

Fig. 1. (A) TEM image of Co nanocrystals synthesized by injecting $0.54 \mathrm{~g}$ of $\mathrm{Co}_{2}(\mathrm{CO})_{8}$ in $3 \mathrm{ml}$ of $o$-dichlorobenzene into $0.1 \mathrm{ml}$ of oleic acid and $0.1 \mathrm{~g}$ of trioctylphosphine oxide in $15 \mathrm{ml}$ of $o$ dichlorobenzene at $455 \mathrm{~K}$. (B, D) TEM images of the cobalt sulfide phases synthesized by injecting sulfur in $o$-dichlorobenzene $(5 \mathrm{ml})$ into cobalt nanocrystal solution with different $\mathrm{Co} / \mathrm{S}$ molar ratios: (B) $\mathrm{Co}_{3} \mathrm{~S}_{4}$ with $\mathrm{Co}: \mathrm{S}=9: 12$ and (D) $\mathrm{Co}_{9} \mathrm{~S}_{8}$ with $\mathrm{Co}: \mathrm{S}=9: 8$. The $\mathrm{Co}_{3} \mathrm{~S}_{4}$ particles were synthesized from the Co sample shown in (A), while the $\mathrm{Co}_{9} \mathrm{~S}_{8}$ particles started from another Co sample which has an average diameter around $11 \mathrm{~nm}$. (C) HRTEM images of $\mathrm{Co}_{3} \mathrm{~S}_{4}$ (left) and $\mathrm{Co}_{9} \mathrm{~S}_{8}$ (right). (E) XRD patterns of cobalt nanocrystals (a) and cobalt sulfide synthesized with different Co/S molar ratios: (b) 9:5; (c) 9:7; (d) 9:8; (e) 9:10; (f) 9:11; (g) 9:12; and (h) 9:18.

Fig. 2. Evolution of $\mathrm{CoO}$ hollow nanocrystals with time by blowing a stream of $\mathrm{O}_{2} / \mathrm{Ar}(1: 4$ in volume ratio, $120 \mathrm{ml} / \mathrm{min}$ ) mixture through a cobalt colloidal solution at $455 \mathrm{~K}$. (A-D) TEM images of the solutions after flowing $\mathrm{O}_{2} / \mathrm{Ar}$ for (A) $0 \mathrm{~min}$; (B) $30 \mathrm{~min}$; (C) $80 \mathrm{~min}$; (D) $210 \mathrm{~min}$. The inset shows a HRTEM of a CoO hollow nanocrystal. (E) XRD patterns of the sample obtained from the solution after flow $\mathrm{O}_{2} / \mathrm{Ar}$ for (a) $0 \mathrm{~min}$; (b) $2.5 \mathrm{~min}$; (c) $5.5 \mathrm{~min}$; (d) $10 \mathrm{~min}$; (e) $30 \mathrm{~min}$; (f) $80 \mathrm{~min}$; and (g) $210 \mathrm{~min}$.

Fig. 3. Evolution of CoSe hollow nanocrystals with time by injection of a suspension of selenium in $o$-dichlorobenzene into a cobalt nanocrystal solution at $455 \mathrm{~K}$, from top-left to bottom-right: 0 $\mathrm{sec}, 10 \mathrm{sec}, 20 \mathrm{sec}, 1 \mathrm{~min}, 2 \mathrm{~min}$ and $30 \mathrm{~min}$. The Co/Se molar ratio is 1:1. 
Fig. 4. (A) Pt nanocrystals prepared by injecting a solution of $0.15 \mathrm{~g}$ platinum acetylacetonate in $5 \mathrm{ml} o$-dichlorobenzene into a refluxing bath of $10 \mathrm{ml} o$-dichlorobenzene containing $0.3 \mathrm{~g}$ of 1,2 hexadecanediol, $0.1 \mathrm{ml}$ of oleic acid, $0.1 \mathrm{ml}$ of oleylamine and $0.06 \mathrm{ml}$ of trioctylphosphine. The solution was then heated for another 120 minutes. (B) Pt@CoO yolk-shell nanostructures formed by injecting $1.08 \mathrm{~g} \mathrm{Co}_{2}(\mathrm{CO})_{8}$ in $6 \mathrm{ml} o$-dichlorobenzene into the Pt nanocrystals solution, and followed by the oxidation of the product particles by blowing a stream of $\mathrm{O}_{2} / \mathrm{Ar}$ (1:4 in volume ratio, $120 \mathrm{ml} / \mathrm{min}$ ) mixture into the colloidal solution at $455 \mathrm{~K}$. The system was kept at the same temperature under stirring for 3 hours. 

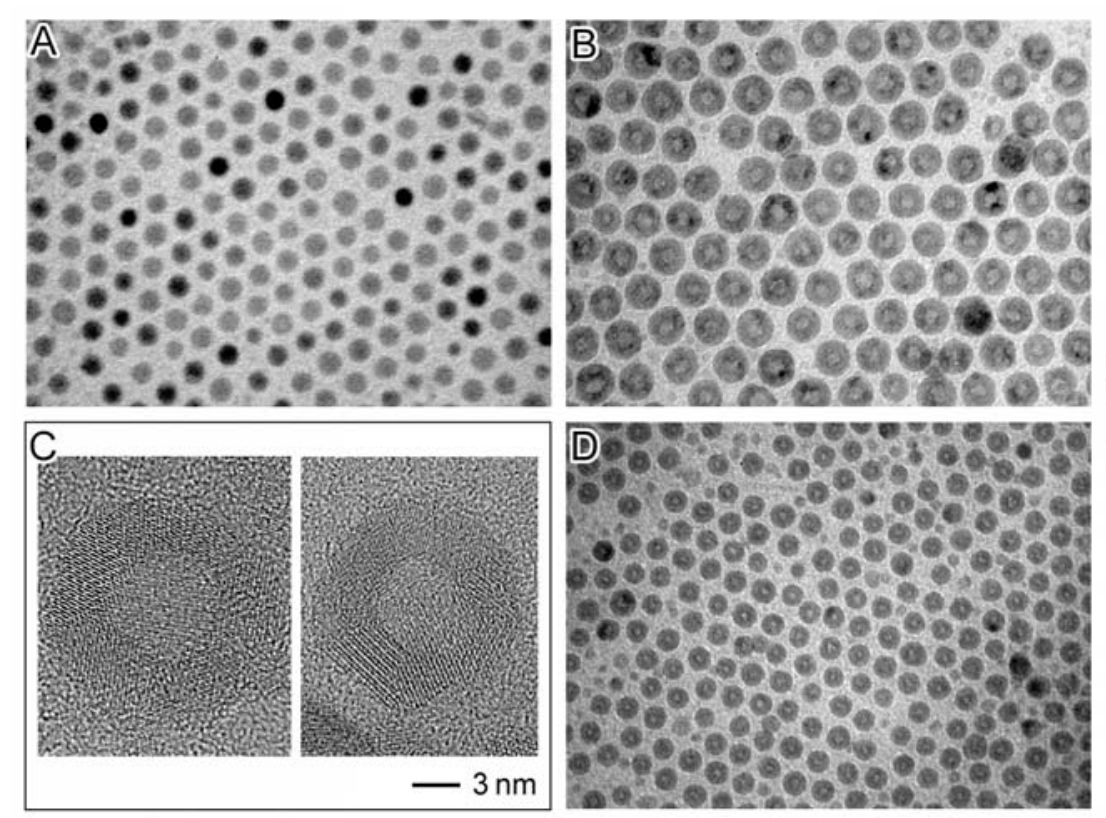

$50 \mathrm{~nm}$

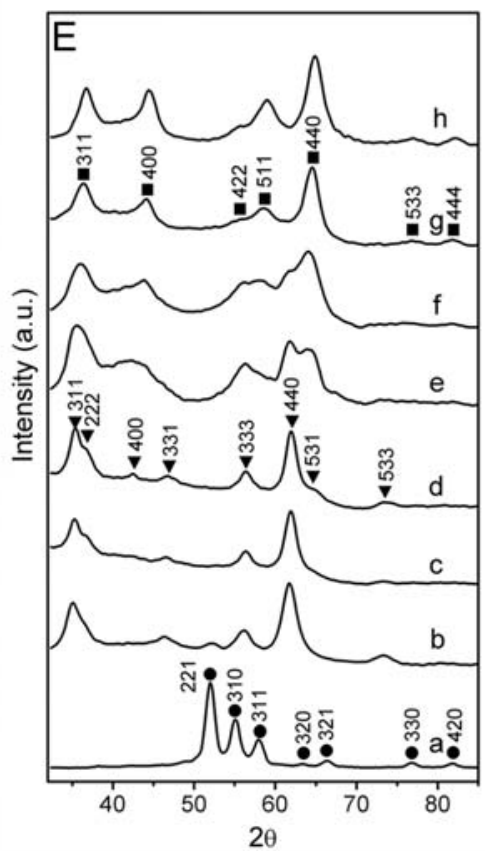

Alivisatos_Fig. 1 

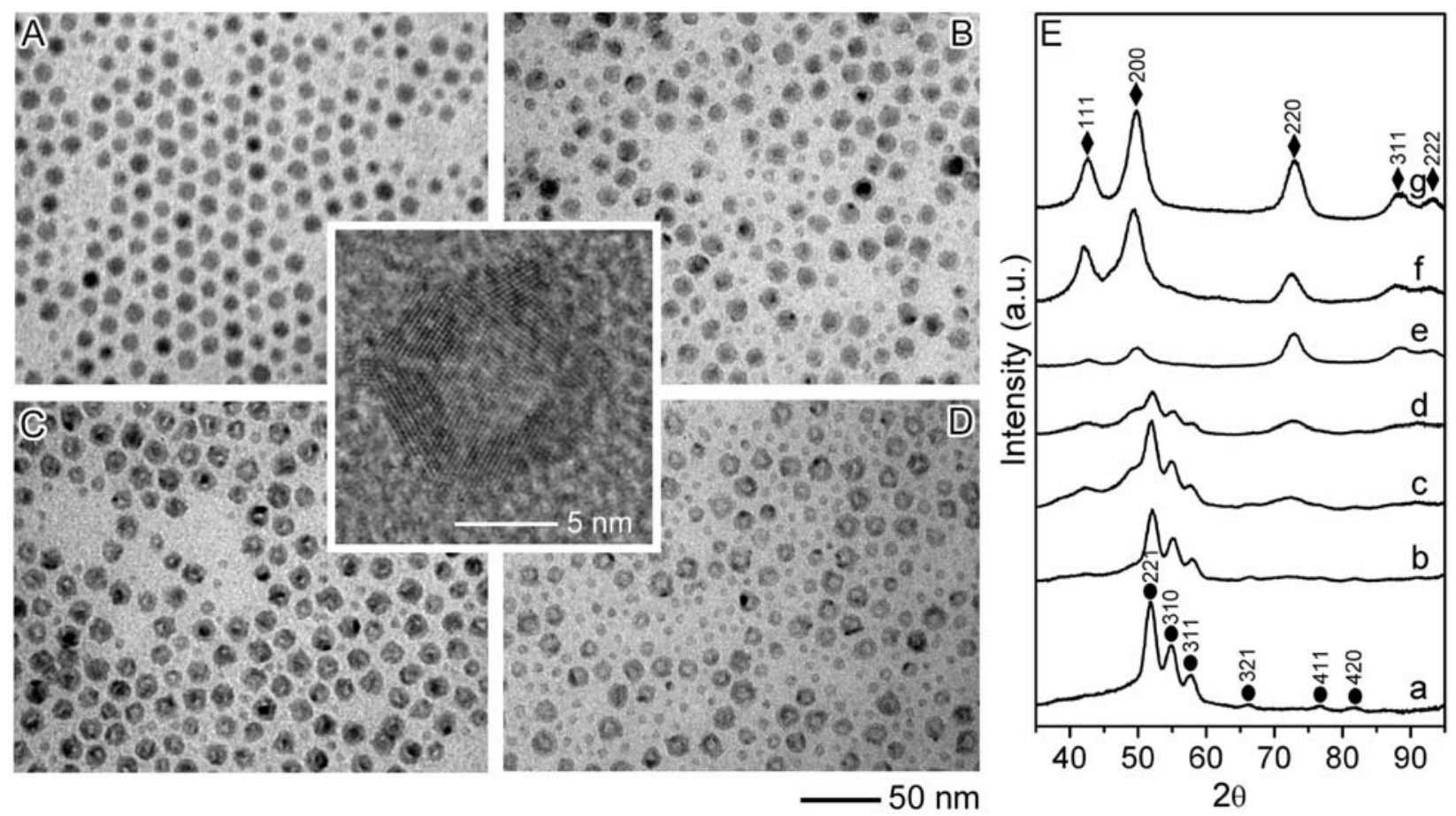

Alivisatos_Fig. 2 

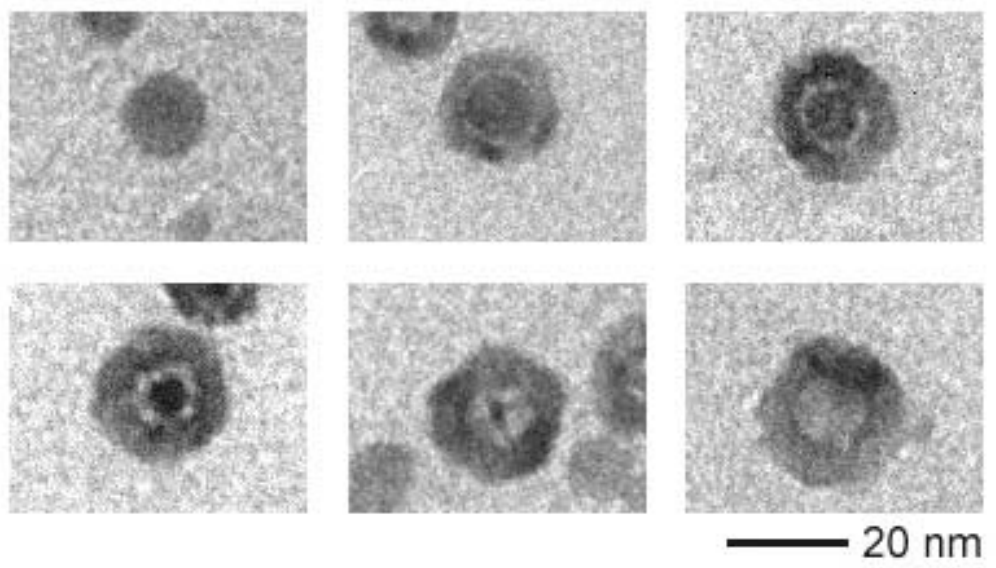

Alivisatos_Fig. 3 

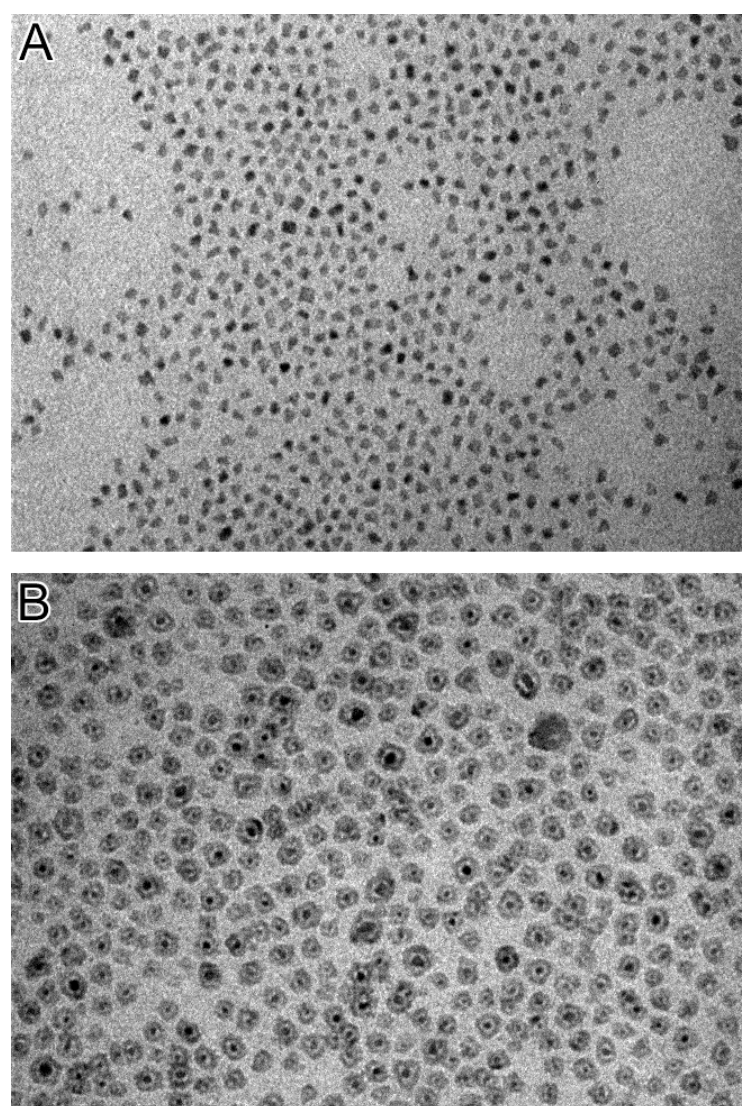
$50 \mathrm{~nm}$

Alivisatos_Fig. 4 